\title{
Co-culture of human nucleus pulposus cells with multipotent mesenchymal stromal cells from human bone marrow reveals formation of tunnelling nanotubes
}

\author{
TOMASZ P. LEHMANN ${ }^{1}$, KRYSTYNA FILIPIAK ${ }^{2}$, WOJCIECH JUZWA ${ }^{3}$, PATRYCJA SUJKA-KORDOWSKA ${ }^{3}$, \\ PAWEŁ P. JAGODZIŃSKI ${ }^{1}$, MACIEJ ZABEL ${ }^{2}$, JAKUB GŁOWACKI ${ }^{4}$, EWA MISTERSKA ${ }^{5}$, \\ MICHAŁ WALCZAK ${ }^{5}$ and MACIEJ GŁOWACKI ${ }^{5}$
}

\begin{abstract}
Departments of ${ }^{1}$ Biochemistry and Molecular Biology, and ${ }^{2}$ Histology and Embryology, Poznan University of Medical Sciences, Poznan 60-781; ${ }^{3}$ Department of Biotechnology and Food Microbiology, Poznan University of Life Sciences, Poznan 60-627; ${ }^{4}$ SSG of Paediatric Orthopaedics and Traumatology, Department of Paediatric Orthopaedics and Traumatology, Poznan University of Medical Sciences, Poznan 61-545; ${ }^{5}$ Department of Paediatric Orthopaedics and Traumatology, Poznan University of Medical Sciences, Poznan 61-545, Poland
\end{abstract}

Received July 13, 2013; Accepted November 1, 2013

DOI: $10.3892 / \mathrm{mmr} .2013 .1821$

\begin{abstract}
Degeneration of the intervertebral disc (IVD) is the main cause of age-related damage of spinal tissues. Using multipotent mesenchymal stromal cells (MSCs) regenerative medicine intends to restore the IVD components of annulus fibrosus (AF) and nucleus pulposus (NP). In the present study NP cells (NPCs) and MSCs obtained from adolescent patients suffering from scoliosis were used. IVDs and vertebrae were obtained during surgery and subsequently processed in order to establish cultures of NPCs and MSCs. The two cell types were co-cultured in $1-\mu \mathrm{m}$ pore size insert system (indirect co-culture) or on one surface (direct co-culture). Prior to co-culture in these systems one of the cell types was stained by lipophilic fluorescent dye DiD (red). The results demonstrated that regardless of the cell type, the flow of DiD from stained to non-stained cells was more efficient in the direct co-culture in comparison with the insert system. Moreover, in the direct system the DiD flow was more efficient from MSCs towards NPCs compared with that in the opposite direction. These data indicated that the membrane interchange between the two cell types was asymmetric. To discriminate the subpopulation of cells that underwent membrane interchange, cells were double stained with DiD and DiO (green). In the first part of the experiment NPCs were stained by $\mathrm{DiO}$ and MSCs by DiD. In the second, NPCs were stained by DiD and MSCs by DiO. The cells were co-cultured in the direct system for 8 days and subsequently analyzed by flow cytometry and confocal microscopy.
\end{abstract}

Correspondence to: Dr Tomasz P. Lehmann, Department of Biochemistry and Molecular Biology, Poznan University of Medical Sciences, ul. 6 Swiecickiego, Poznan 60-781, Poland

E-mail: tlehmann@ump.edu.pl

Key words: nucleus pulposus, multipotent mesenchymal stromal cells, intervertebral disc, tunnelling nanotubes, DiO, DiD
This analysis revealed that $>50 \%$ of cells were stained by the $\mathrm{DiO}$ and DiD dyes. NPCs and MSCs formed structures similar to tunnelling nanotubes ( $\mathrm{TnT}$ ). In conclusion, the formation of TnT-like structures is able to promote, phenotypic changes during the direct co-culture of NPCs with MSCs.

\section{Introduction}

The adult human intervertebral disc (IVD) consists of an extracellular matrix combined with a small number of cells. The external part of the IVD is composed of an annulus fibrosus (AF) that surrounds the nucleus pulposus (NP) in the centre. The IVD possesses low regeneration capabilities mainly due to the avascular system of metabolite transport. NP damage is usually irreversible, therefore numerous attempts have been undertaken to stimulate regeneration of the nucleus pulposus cells (NPCs) (1).

Studies on the interaction between the IVD and multipotent mesenchymal stromal cells (MSCs) have been performed in human and animal culture systems (2-4). Direct co-culture between human MSCs and human AF cells was required to observe an increase in the glycosaminoglycan (GAG) content (5). The increase of mRNA of the NP marker genes COL2A1, COL6A2, ACAN and SOX9 has been reported as the main result of direct co-culture of human NPCs and MSCs (6). Indirect co-culturing of NPCs and MSCs in the insert system promoted COL2A1 expression in the MSCs and the proliferation of NPCs as well as the expression of $A C A N$ (7). A study of human MSCs that were co-cultured with NP revealed higher expression of COL2A1 and ACAN (8). Direct co-culture has been reported as the most efficient way of inducing the NP-like phenotype in MSCs observed as an increase in SOX9, COL4 and $A C A N$ expression including growth factor production (9). Degenerate NPCs regained a non-degenerate NP phenotype as a result of close contact with MSCs (9).

It has recently been demonstrated that cell fusion or gap-junction are a marginal form of intercellular communication 
between NPCs and MSCs (10). It was revealed that NPCs and MSCs exchanged CFDA and SNARF hydrophilic dyes and after 7 days $0.75 \%$ of the cells were hybrids of NPCs and MSCs (10). By contrast, the lipophilic dye DiI was transported from labeled to unlabeled cells much more efficiently and after 7 days of co-culture $80 \%$ of the unlabeled cells were stained (10). The amount of microvesicles (MVs) detected in the culture media collected from the DiI-stained cells was insufficient to explain such intensive membrane transfer (10). Tunnelling nanotubes (TnTs) are structures connecting two cells of the same phenotype, different phenotypes or tissues. Their diameter ranges from 50 to $200 \mathrm{~nm}$ and may reach several cell diameters long. TnT structures are present in various types of cells including fibroblasts, neurons, immune cells and cancer cells (11). An increasing number of data have demonstrated that TnTs are highways of intercellular transport (11). Findings of previous studies (12) have demonstrated TnT in various cell types, nevertheless, there are no studies demonstrating TnT in NP-MSC co-cultures.

In the present study NPCs and MSCs were obtained from patients with scoliosis. The cells were then co-cultured in common medium on separate surfaces (called: non contact, indirect or insert system) and with one-surface conditions (called direct system). Using a cell sorter and a confocal microscope human NPCs and MSCs were found to create protrusions similar to TnTs. In these tubes a reciprocal exchange of lipophilic dyes DiO (green) and DiD (red) occurred. This exchange was more efficient between cells growing in direct co-culture in comparison with the porous membrane (insert system).

The present study focused on the issue of determining which type of structure is required for the direct interaction of NPCs and MSCs. The objective of this study was to discriminate the subpopulation of cells that underwent membrane interchange using $\mathrm{DiO}$ and DiD dyes and to verify the hypothesis that NP and MS cells are able to establish contact channels to exchange components of membranes.

\section{Materials and methods}

Patients and tissues. Human NPCs were collected using an anterior approach from 12 patients undergoing treatment to correct thoraco-lumbar or lumbar scoliosis during routine preparation of the site for anterior spodylodesis. All the patients were recruited into the study consecutively.

The eligibility criteria for the study were: i) adolescent idiopathic scoliosis; ii) 10-19 years of age; iii) a Cobb angle of over $40^{\circ}$ and iv) scoliosis correction from an anterior approach with routine removal of an IVD in preparation of the site for anterior spodylodesis. This process complies with ethical considerations with regard to the collection of human NPCs. NPCs extracted from the IVDs of adolescent subjects were presumed to be non-degenerative.

The exclusion criteria were: i) use of analgesic, antibiotic or steroid medication prior to hospital admittance and ii) previous surgery in the spinal area.

Patients who fulfilled the inclusion criteria received in-depth information on the aim of the study and were assured of anonymity. Informed consent from the legal guardians of each patient was obtained prior to the request to collect NPCs from the donors was made. The mean \pm SD age of the patients was $16 \pm 2.3$ years (range 14-19). The study design was approved by the Ethics Committee of Poznan University of Medical Sciences (Poznan, Poland; approval no. 838/09) and was carried out in accordance with universal ethical principles.

\section{Cell culture}

Nucleus pulposus. Non-degenerate IVD tissue was dissected mainly from Th12 to L3 to separate the NP from the AF tissue. The NP was enzymatically digested overnight at $37^{\circ} \mathrm{C}$ with $0.02 \%$ collagenase type II (Sigma, St. Louis, MO, USA) in serum-free medium containing 100 units penicillin, $100 \mu \mathrm{g}$ streptomycin and $250 \mathrm{ng}$ amphotericin B per ml (ABAM, Sigma). The digested tissue/cell suspension was filtered through a sterile nylon fabric to remove remaining tissue debris. The cells were centrifuged at $300 \mathrm{~g}$ for $5 \mathrm{~min}$, seeded onto a tissue culture flask and cultured at $37^{\circ} \mathrm{C}$ in $5 \% \mathrm{CO}_{2} / 95 \%$ air, in $(1: 1$ v/v) Dulbecco's modified Eagle's medium/nutrient F-12 Ham supplemented with $10 \%$ fetal bovine serum, ABAM (all from Sigma) solution and vitamin C (5 mg/ml). For gene expression experiments, 6 -well plates were used and 150,000 cells were placed in each well.

Bone marrow. Non-degenerative vertebrae were extracted mainly from Th12 to L3. The vertebrae were minced mechanically and enzymatically digested overnight in similar conditions as for the NPCs. The digested tissue/cell suspension was filtered and resuspended in $1 \mathrm{ml}$ PBS buffer and $20 \mu \mathrm{l}$ fetal bovine serum (Sigma). Subsequent steps of stem cell enrichment were performed according to the stem cells kit procedure (RosetteSep Human Mesenchymal Stem Cell Enrichment Cocktail, StemCell Technologies, Vancouver, $\mathrm{BC}$, Canada). Briefly, to the bone marrow cells resuspended in $1 \mathrm{ml}$ PBS buffer and $20 \mu \mathrm{l}$ fetal bovine serum $50 \mu \mathrm{l}$ of RosetteSep Human Mesenchymal Stem Cell Enrichment Cocktail (StemCell Technologies) was added. Subsequent to a 20 min incubation the cells were placed in the ficoll solution and centrifuged. The intermediate layer of the cells was transferred into a cell culture dish and placed in the incubator in a medium similar to the one used for the NPCs except that in this case the culture medium, Dulbecco's modified Eagle's medium/nutrient F-12 Ham, was not enriched with vitamin C. For the first 4 days of culture the cells were washed daily to enable the selection of adhesive cells only. The MSCs obtained were cultured in a chondrocyte selective cell culture medium (Human Mesenchymal Stem Cell Functional Identification Kit, R\&D Systems Inc., Minneapolis, MN, USA). The MSC phenotype was confirmed by quantitative reverse transcription PCR (RT-qPCR). MSCs destined for co-culture with NPCs were stained with lipophilic dyes and subsequently cultured in the insert system or in direct co-culture with NPCs. The MSCs were analysed with a flow cytometer (BD FACSAria ${ }^{\mathrm{TM}}$ III; Becton Dickinson, Franklin Lakes, NJ, USA) and confocal laser scanning microscope (Olympus Fluoview FV10i; Olympus, Tokyo, Japan).

Staining cells for flow cytometry and confocal microscopy. For flow cytometry and confocal microscopy the cells were cultured and stained with lipophilic dyes DiO (green) or DiD (red) (Molecular Probes, Inc., Eugene, OR, USA). The cells were removed from the culture dish using trypsin (Sigma), in accordance with the manufacturer's instructions, and 
Table I. Primers used in quantitative PCR.

\begin{tabular}{|c|c|c|c|}
\hline Gene & Primer sequence & Amplicon length, bp & GenBank accession nos \\
\hline$A C A N$ & $\begin{array}{l}\text { 5'-ACCAGACTGTCAGATACCCC-3' } \\
\text { 5'-CATAAAAGACCTCACCCTCC-3' }\end{array}$ & 156 & NM_001135 \\
\hline SOX9 & $\begin{array}{l}\text { 5'-GAAGAACGGGCAGGCGGA-3' } \\
\text { 5'-TTTGGGGGTGGTGGGTGG-3' }\end{array}$ & 181 & NM_000346 \\
\hline COL2Al & $\begin{array}{l}\text { 5'-ACCAGGACCAAAGGGACA-3' } \\
\text { 5'-GCAGCAAAGTTTCCACCA-3' }\end{array}$ & 246 & NM_033150 \\
\hline COL1A1 & $\begin{array}{l}\text { 5'-GAAGGGACACAGAGGTTTCAG-3' } \\
\text { 5'-TTCCACGAGCACCAGCAG-3' }\end{array}$ & 179 & NM_000088 \\
\hline$T G F B 1$ & $\begin{array}{l}\text { 5'-GAAACCCACAACGAAATC-3' } \\
\text { 5'-AATTTCCCCTCCACGGCT-3' }\end{array}$ & 300 & NM_000660 \\
\hline MRPL19 & $\begin{array}{l}\text { 5'-TCCAACCGCCGCCGAAAC-3' } \\
\text { 5'-AACACGAAGAATACTTCCAACA-3' }\end{array}$ & 197 & NM_014763.3 \\
\hline$H M B S$ (PBGD) & $\begin{array}{l}\text { 5'-CCCTGGAGAAGAATGAAGTG-3' } \\
\text { 5'-TCCCCGAATACTCCTGAA-3' }\end{array}$ & 254 & NM_000190 \\
\hline
\end{tabular}

subsequently counted. The required number of cells were incubated for $30 \mathrm{~min}$ with $10 \mu \mathrm{l}$ of $\mathrm{DiO}$ or DiD dye solutions in $1 \mathrm{ml}$ of cell suspension in PBS. The cells were subsequently washed with PBS and cultured overnight in a culture medium appropriate to the cell type. The following day the stained cells were subcultured to the new culture dishes depending on the experiment requirements.

NPCs and MSCs were stained with DiD, cultured in the $1-\mu \mathrm{m}$ insert system (Cell Culture Inserts for 6-well plates, $1 \mu \mathrm{m}$, translucent PET membrane, BD Falcon ${ }^{\mathrm{TM}}$, Franklin Lakes, NJ, USA) and placed in culture dishes (6-well Cell Culture Plate, BD Falcon ${ }^{\mathrm{TM}}$ ). In the 6-well culture dishes the same number of non-stained MSCs and NPCs were cultured, respectively, under the inserts. In an alternative version of this experiment NPCs and MSCs were stained with DiD and cultured together with non-stained MSCs or NPCs respectively in 6-well culture dishes at a ratio of 50:50. Following 4 days of culture, the cells were detached from the dishes by trypsin and then analysed using the BD FACSAria ${ }^{\mathrm{TM}}$ III (Becton Dickinson).

The cells designated for the flow cytometry in the double staining system were stained with $\mathrm{DiD}$ or $\mathrm{DiO}$ and cultured in 6-well culture dishes at a ratio of 50:50. The experiment was performed in two versions, the first with NP/DiO and MSC/DiD and the second with NP/DiD and MSC/DiO. Following 8 days of culture the cells were detached from the dishes by trypsin, sorted on BD FACSAria III and the subsequent double-stained population was cultured again. For laser confocal microscopy the cells were cultured on glass bottom dishes (3.5 cm diameter) from Mattek (Ashland, MA, USA).

Confocal and fluorescent microscopy. The Olympus Fluoview FV10i confocal laser scanning microscope with the 60x water immersion objective lens (NA=1.35) was used (Olympus). Images were captured in two fluorescence channels: DiO, DiD and in the phase-contrast channel. The images were prepared using FV10-ASW software (Olympus). Images from the three channels were combined into one.

Flow cytometric analysis combined with cell sorting. Culture cells were digested with $1 \mathrm{X}$ trypsin and analyzed using a BD FACSAria $^{\mathrm{TM}}$ III (Becton Dickinson) flow cytometer (cell sorter), equipped with four lasers $(375,405,488$ and $633 \mathrm{~nm})$, 11 fluorescence detectors, forward scatter (FSC) and side scatter (SSC) detectors. Side scatter signals and fluorescent light was collected by a gel-coupled, NA 1.2 collection lens. The instrument setup (optical alignment), stability and performance test was performed using the Cytometer Setup and Tracking system by Becton Dickinson. FACSFlow solution (Becton Dickinson) was used as a sheath fluid. The configuration of the flow cytometer included a $100 \mu \mathrm{m}$ nozzle and 20 psi sheath fluid pressure. The cells were characterized by the non-fluorescent parameters FSC and SSC, and the fluorescent parameters green fluorescence from $\mathrm{DiO}$ reagent collected using a 530/30 band pass filter and red fluorescence from the DiD reagent collected using a 660/20 band pass filter. The 488 and $633 \mathrm{~nm}$ lasers were employed in excitation of $\mathrm{DiO}$ and $\mathrm{DiD}$ fluorescent reagents, respectively. Flow cytometric analyses were performed using logarithmic gains and specific detector settings. The threshold was set at the FSC signal. Data were obtained in a four-decade logarithmic scale as area signals and analyzed with the FACS DIVA software (Becton Dickinson). The populations were defined by gating in the dot plots of $\mathrm{DiO}$ versus DiD. Each sample was analyzed in triplicate. Cell sorting preceded the doublet discrimination procedure with the use of height versus width scatter signal measurements, in order to discriminate single cells from conglomerates. The cells were sorted in $5 \mathrm{ml}$ cytometric tubes.

Glycosoaminoglycans. NPCs were grown in appropriate culture media, then digested with papain following the indicated time elapsed. Subsequently, Blyscan Sulfated Glycosaminoglycan 

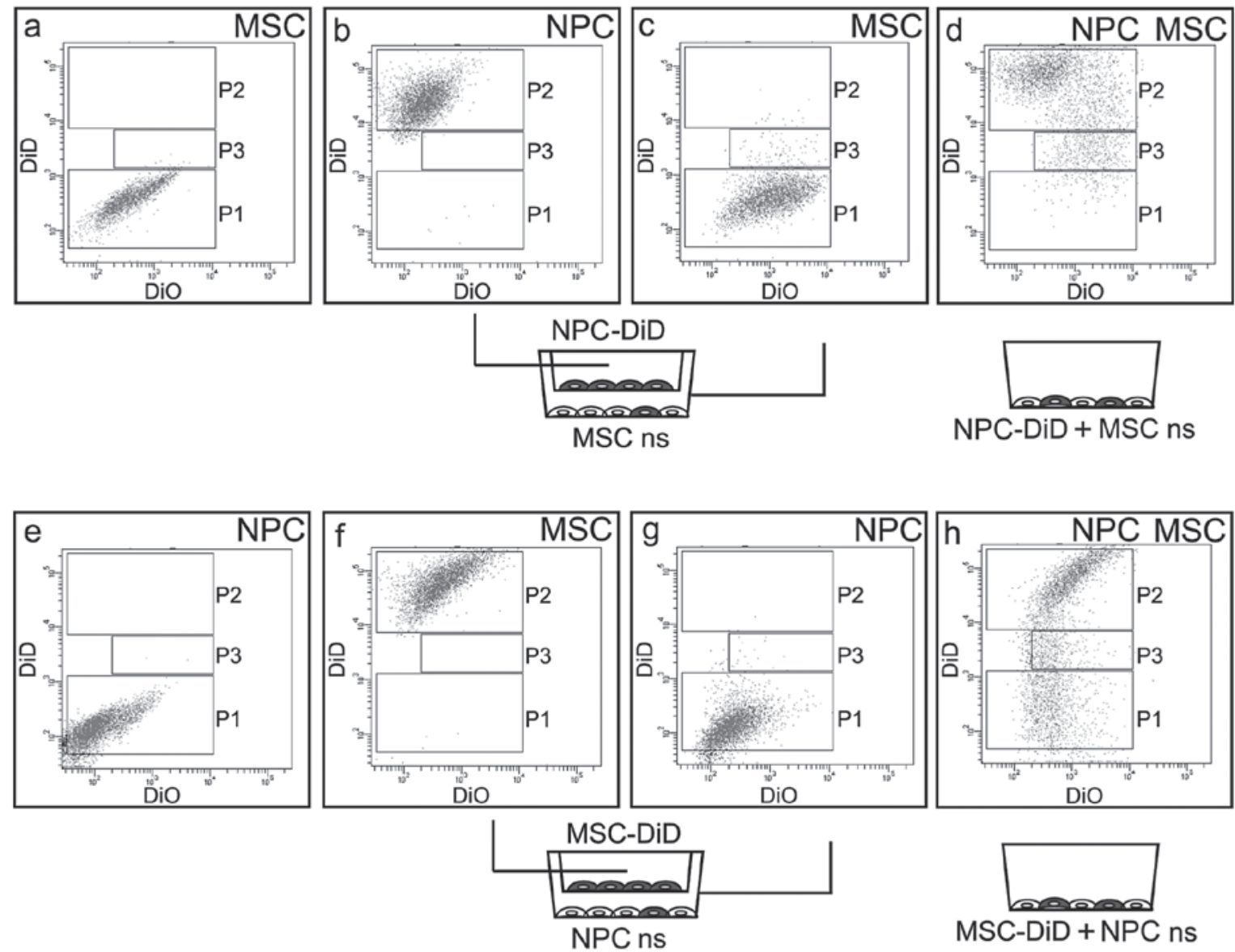

Figure 1. Flow cytometric results obtained following analysis of NPCs and MSCs stained with DiD. The results obtained are subsequent to 4 days of culture and analysed by a flow cytometer. (a) Analysis of control MSCs non-stained (ns) which were cultured for 4 days without contact with NPCs. (b) Analysis of NPCs prestained with DiD and co-cultured over non-stained MSCs in the upper dish of a 1- $\mu \mathrm{m}$ insert system. (c) Analysis of non-stained MSCs cultured under DiD prestained NPCs in the lower dish of a 1- $\mu$ m insert system. (d) Analysis of one surface co-cultured DiD prestained NPCs and non-stained MSCs. (e) Analysis of control NP non-stained (ns) which were cultured for 4 days without contact with MSCs. (f) Analysis of MSCs prestained with DiD and co-cultured over non-stained NPCs in the upper dish of a 1- $\mu \mathrm{m}$ insert system. (g) Analysis of non stained NPCs cultured under DiD prestained MSCs in the lower dish of a 1- $\mu \mathrm{m}$ insert system and (h) analysis of co-cultured DiD prestained MSCs and non-stained NPCs. NPCs, nucleus pulposus cells; MSCs, mesenchymal stromal cells.

Assays (Biocolor Ltd., Carrickfergus, UK) were performed according to the manufacturer's instructions.

Gene expression. The NP total RNA was extracted from the cultured cells with the use of TRItidy $\mathrm{G}$, according to the manufacturer's instructions (Applichem, Darmstadt, Germany). In total, $1 \mu \mathrm{g}$ total RNA was reverse-transcribed using a Superscript Reverse Transcriptase Kit (Life Technologies, Carlsbad, CA, USA). Oligo(dT) $)_{15}$ primed cDNAs were amplified by qPCR, using the primers listed in Table I, and the LightCycler FastStart DNA Master SYBR-Green I kit (Roche, Mannheim, Germany). The crossing-point values were calculated automatically based on the second derivative algorithm, and the results were analyzed by the relative expression method. HMBS and MRPL19 were used as reference genes.

\section{Results}

Cell line identity. The NPCs obtained surgically were cultured and the mRNA was subsequently extracted. Reverse transcription and PCR revealed the expression of COL1A1, COL2A1, $T G F B 2, S O X 9$ and $A C A N$ (results not shown). In addition, the GAG levels in cultures were measured using the Blyscan kit (results not shown) and the presence of GAG in the cultured cells was detected.

MSCs were selected from total bone marrow cells by their ability of adhesion to a plastic surface. This method is commonly used to obtain multipotent MSCs (13). The cells were cultured in a chondrogenesis-stimulating medium to control their differentiation potential. MSCs cultured in the medium stimulating chondrocyte differentiation revealed increased levels of mRNA of SOX9, TGFBI and ACAN in correlation to MSCs cultured in the standard medium (data not shown). TGFB1, transforming growth factor $\beta 1$ gene, is responsible for MSC differentiation and is one of the main components of chondrocyte differentiation media. ACAN aggrecan gene encodes the protein component of proteoglycans. SOX 9 is a gene of transcription factor SOX-9 and is important in chondrocyte differentiation. These experiments confirmed the differentiation potential of our primary MSC lines.

Differences in the DiD transport between the NPCs and MSCs in two systems of co-culture. NPCs and MSCs were stained with DiO and DiD lipophilic dyes and cultured in the following combinations: NP/DiO with NP/DiD and MSC/DiO 

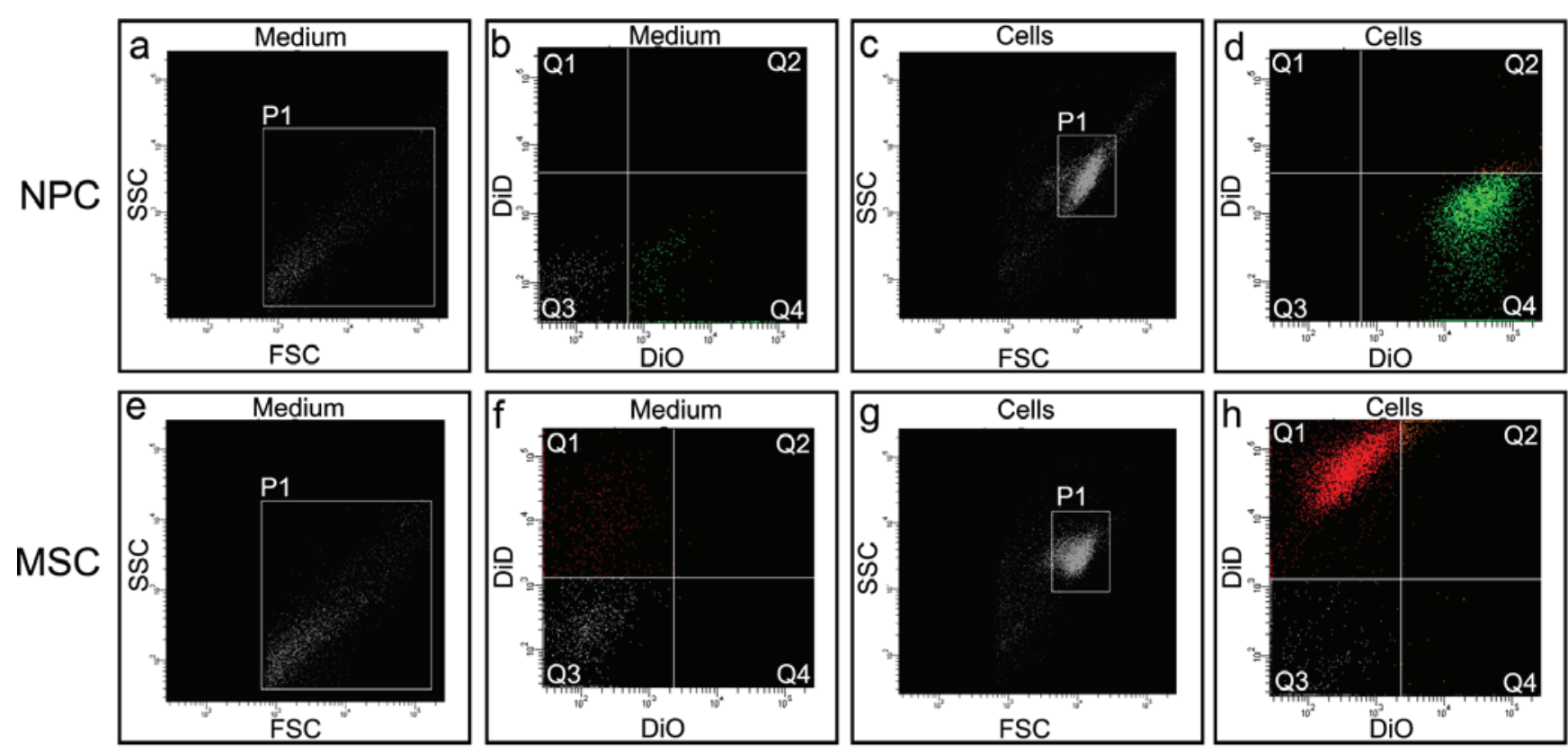

Figure 2. Flow cytometric results obtained subsequent to analysis of the media from over NPCs and MSCs stained with DiO and DiD, respectively. Subsequent to 2 days of co-culture the cell media were measured in the flow cytometer. Forward (FSC) and side scatters (SSC) including the fluorescence diagrams are shown. Stained NPCs and MSCs are also shown for comparison. (a) Side versus forward scatter analysis of medium from over NPCs prestained with DiO which were cultured without contact with MSCs. (b) Analysis of fluorescence of the medium from over NPCs prestained with DiO. (c) Side versus forward scatter analysis of NPCs prestained with DiO which were cultured without contact with MSCs. (d) Analysis of fluorescence of the NPCs prestained with DiO. (e) Side versus forward scatter analysis of medium from over MSCs prestained with DiD which were cultured without contact with NPCs. (f) Analysis of fluorescence of the medium from over MSCs prestained with DiD. (g) Side versus forward scatter analysis of MSCs prestained with DiD which were cultured without contact with NPCs and (h) analysis of fluorescence of the MSCs prestained with DiD. MSCs, mesenchymal stromal cells; NPCs, nucleus pulposus cells.

with MSC/DiD. The two co-cultures revealed exchange of dyes following $24 \mathrm{~h}$ of culture (data not shown).

The comparison of DiD transport in two co-culture systems between NPCs and MSCs was measured by flow cytometry. The first system contained a culture dish and a $1-\mu \mathrm{m}$ pore insert. The second culture system was a one surface co-culture. DiD prestained NPCs were cultured in the insert and non-stained MSCs were cultured with under-stained NPCs. Following 4 days of co-culture the two cell lines underwent flow cytometric analysis. The stained NPCs were detected and are marked as P2 in the diagram (Fig. 1b). The population of the MSCs (P1) was grouped in the region corresponding to the population of the non-stained control ( $\mathrm{P} 1$ in Fig. 1a, $\mathrm{P} 1=99.0 \%, \mathrm{P} 2=0.0 \%$ and $\mathrm{P} 3=0.5 \%$ and Fig. $1 \mathrm{c}, \mathrm{P} 1=96.4 \%, \mathrm{P} 2=0.4 \%$ and $\mathrm{P} 3=2.7 \%$ ), however, this population was slightly diffused and the subpopulation P3 was increased. (Fig. 1c). The subpopulation P3 revealed intermediate intensity of the DiD signal between non-stained MSCs and DiD-stained NPCs (population P2). The co-culture of prestained NPCs with non-stained MSCs on the direct system revealed a population corresponding to DiD-stained NPCs (P2) (Fig. 1d, P1=5.2\%, P2=69.0\% and P3=14.5\%). The subpopulation of intermediately stained cells (P3) was more numerous compared with the corresponding populations in the indirect system (14.5 vs. 2.7\%). The population P1 corresponding to non-stained MSCs was almost absent. Thus, the subpopulation P3 is likely to be mainly an MSC population, stained by DiD dye absorbed from the prestained NPCs. Moreover, the dye exchange in the direct, one-surface system is more efficient compared with the indirect co-culture (insert system).
To compare the cell lines in their DiD exchange efficiency, MSCs were also stained with DiD and were co-cultured with non-stained NPCs. DiD prestained MSCs were cultured in the insert and non-stained NPCs were cultured with under-stained MSCs. Following 4 days of co-culture the two cell lines were analyzed by a flow cytometer. The stained MSCs were detected and marked as $\mathrm{P} 2$ in the diagram (Fig. 2f, $\mathrm{P} 1=0.1 \%, \mathrm{P} 2=96.7 \%$ and $\mathrm{P} 3=0.0 \%)$. The dominant population of NPCs (P1) was grouped in the region corresponding to the population of the non-stained control (Fig. 1e,P1=84.9\%,P2=0.0\% and P3=0.0\%), however, this population was diffused and the subpopulation $\mathrm{P} 3$ was discriminated (Fig. $1 \mathrm{~g}, \mathrm{P} 1=93.9 \%, \mathrm{P} 2=0.1 \%$ and $\mathrm{P} 3=0.5 \%$ ). The subpopulation P3 revealed an intermediate intensity of DiD signal between non-stained NPCs and DiD-stained MSCs. Co-culture of prestained MSCs (P2) with non-stained NP (P1) in the direct system revealed a great population corresponding to the DiD-stained MSCs (P3) (Fig. 1h, P1=29.5\%, P2=51.3\% and $\mathrm{P} 3=12.6 \%)$. The subpopulation of intermediately stained cells (P3) was greater than the corresponding population in the insert system (12 vs. $0.5 \%)$. The population P1 corresponding to non-stained NPCs was present (Fig. 1e). We hypothesized that the subpopulation P3 is likely to be mainly an NP population that was stained by DiD dye absorbed from prestained MSCs. By comparing the populations P1 (non-stained MSCs) and P1 (non-stained NPCs) in Fig. 1d and h, respectively, it was found that in the one-surface system with direct contact of cells, the flow of the DiD dye was more efficient from MSCs towards NPCs compared with that in the opposite direction.

$M V$ detection. To verify the hypothesis that a flow of particles between the cells co-cultured in the insert system exists a 

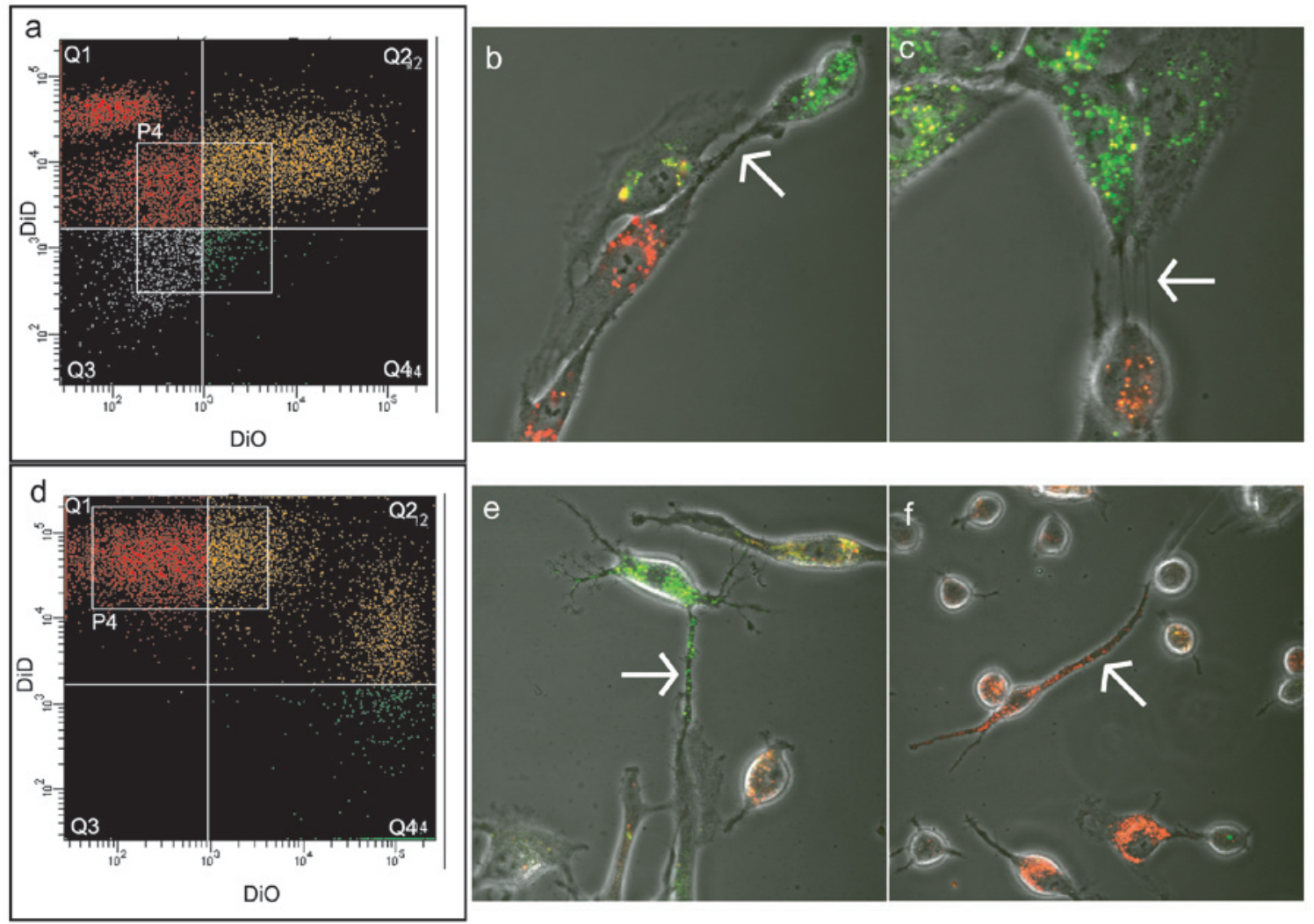

Figure 3. Flow cytometric analysis and confocal microscopy images of NPCs and MSCs stained with (a-c) DiO and DiD respectively or (d-f) DiD and DiO. Co-cultured NP-MSCs were subsequently separated by (a and d) BD FACSAria ${ }^{\mathrm{TM}}$ III. (a) Population P4 from the NP/DiO and (d) MSC/DiD experiment and population $\mathrm{P} 4$ from the MSC/DiO and NP/DiD experiments were selected for further culture. The two cultures were analyzed under a confocal microscope subsequent to 4 days in culture. The arrows show thin and thick TnT-like structures. NPCs, nucleus pulposus cells; MSCs, mesenchymal stromal cells; TnT, tunelling nanotubes.

cytometric analysis of the culture media was performed. Two possible paths of lipophilic dye transport were considered: free dye diffusion or transport in secreted microvesicles. The culture media from NPCs prestained with DiO or MSCs prestained with DiD were collected subsequent to 2 days of culture. A flow cytometric analysis of the cell culture medium from $\mathrm{DiO}$ prestained $\mathrm{NP}$ revealed the presence of $\mathrm{DiO}$-stained particles (Fig. 2b, Q4) of a diameter smaller than the NPCs (Fig. 2a vs. c, P1). The cytometric analysis of the cell culture medium from over DiD-prestained MSCs revealed the presence of DiD stained particles (Fig. 2f, Q1) of a diameter smaller than that of an MSC (Fig. 2e vs. g, P1).

Cell-to-cell communication in direct co-culture of NPCs and MSCs. Prestained NP/DiO with MSC/DiD were cultured at a ratio of $1: 1$ on $3.5 \mathrm{~cm}$ diameter culture dishes for 8 days. In a parallel alternative experiment the same amount of $\mathrm{MSC} / \mathrm{DiO}$ and NP/DiD was cultured in $3.5 \mathrm{~cm}$ culture dishes for 8 days. Co-cultured NP-MSC cells were then separated on the BD FACSAria III. The distribution of dyes was asymmetric (Fig. 3a and d) and therefore it was concluded that the NP-MSC communication is likely to be asymmetric. The separated population P4 (Fig. 3a) from the NP/DiO and MSC/DiD experiment was selected for subsequent culture. The population $\mathrm{P} 4$ (Fig. 3d) from the MSC/DiO and NP/DiD experiment was also selected for further culture. The two cultures were analyzed by confocal microscopy subsequent to 4 days in culture and revealed exchange of $\mathrm{DiO}$ and $\mathrm{DiD}$ between the two cell lines in the post-sorting culture (Fig. 3b and c, e and f). Analysis of the cells stained by confocal microscopy by NP/DiO and MSC/DiD including NP/DiD and MSC/DiO revealed that 4 days of culture is sufficient to enable detection of at least two types of cell structures that may be responsible for lipophilic dye transport. Thin and thick linear structures that connect NPCs and MSCs were visible (arrows in Fig. 3b, c, e and f). It may be that the thin linear structures are pre-TnTs, which later expand to form thicker communication arteries. Different phases of exchange in the two dyes are also visible: from clear green through light green to orange and yellow and from deep red through light red to orange and yellow.

\section{Discussion}

It has previously been revealed that the exchange of cellular membranes between NPCs and MSCs is dependent on MVs (10). It has been indicated that the intensity of lipophilic dye exchange requires other structures including $\operatorname{TnTs}(10,12)$. In the present study it was confirmed that when NPCs and MSCs were grown on one surface (direct co-culture) the exchange of lipophilic dye (DiD) was more efficient in comparison with the transport mediated by the culture medium in the insert system. Two types of structures were observed similar to TnTs formed between NPCs and MSCs grown on one surface. The high rate of lipophilic dye transport between the cells in culture 
on one surface in comparison with co-culture in the insert system is the additive effect; on the one hand engaging known phenomena of gap junctions, cell fusion and MV release, and on the other, the transient formation of TnTs.

Therapies based on cells obtained from patients, cultured and subsequently reintroduced into the patient's body may be used in the treatment of IVD degeneration (14). Studies performed in animal and human cells have indicated the possibility of reciprocal stimulation of differentiated IVD cells and MSCs $(2,5)$. Although this has been known for at least a decade, little is known regarding the molecular mechanisms of cell-to-cell communication. MSCs from various sources, mainly bone marrow, were used for the development towards a chondrocyte-like phenotype (6). The International Society for Cellular Therapy recommends applying the term 'multipotent MSCs' to the fibroblast-like plastic-adherent cells (13). However, the same organization also indicates that the term MSCs may be used. To promote differentiation of animal or human MSCs, several methods were developed which involved growth factors from the transforming growth factor family (15-18). An alternative approach is the co-culture of IVD cells with MSCs in the 'one-surface system' (also known as direct co-culture or contact system), three-dimensional systems (alginate beads) and culture of two cell lines separated by a porous membrane (also termed insert system, indirect co-culture or non-contact system) (19). Injection of MSCs into the disc space was also assayed $(16,20)$.

The superiority of direct co-culture of NPCs and MSCs over the indirect systems has been observed in earlier studies demonstrating the modulation of gene expression in NPCs and MSCs in a direct co-culture system (9). In the experiments of the present study, non-stained MSCs were co-cultured with DiD-stained NPCs, or DiD-stained MSCs with non-stained NPCs. This approach revealed that the culture medium is a path for lipophilic dye transport and non-stained cells adsorbed DiD from stained cells. In comparison with the insert system, co-culture on one surface caused a relatively higher exchange of the lipophilic dye between NPCs and MSCs. Similar observations have been made in other cell lines. PKH67-labeled $\mathrm{CHO}$ and CD36+ cells were cultured using a $0.4 \mu \mathrm{m}$ insert system and the one-surface system. In comparison with the one-surface, the transfer of proteins and lipids was much lower in the insert system (21). The abovementioned results and those of the present study provide strong evidence that a type of additional means of transport occurs during a one-surface co-culture, known as the spontaneous intercellular transfer of cellular components (21). As a result of membrane contact, the outer phospholipid bilayers may provoke a transient fusion called hemifusion. Subsequently, the two membranes might locally form fusion pores or activation of fusogenic proteins leading to complete cell-cell fusion $(21,22)$.

To determine whether or not the culture medium contained particles detectable in the cytometric analysis the media from DiD-stained cells were analyzed. Stained structures smaller than the cells were detectable in the media and may be responsible for indirect cell contact. This observation is similar to earlier data from the indirect co-culture system of NPCs and MSCs stained by DiI (10). DiI was exchanged in a more intense way, between cells cultured in the direct system in correlation to the insert indirect system (10). It has been indicated that in addition to $\mathrm{MV}$, alternative ways of cell contact occur in the direct co-culture (10).

The NPCs and MSCs were analysed in a double-stained co-culture system. In the images from the confocal microscopy of NPCs and MSCs stained with DiO and DiD, respectively, we observed an intense exchange of dyes. The majority of the double-stained cells were not larger than a single NPCs and MSCs. However, there was a small fraction of doublets that may indicate the existence of hybrids. A small amount of double-stained cells were grown again in a one-surface culture system revealing thin protrusions connecting the cells. Several of these structures were stained, thus that the exchange of dyes via this structure is possible.

Several studies (2-10) have been undertaken to induce a reciprocal stimulation of animal NPCs and MSCs in a co-culture system. In experiments using rabbit cells, NPCs and MSCs were grown on opposite sites of the same $0.4 \mu \mathrm{m}$ pore size membrane (insert system) where direct contact of cells was possible (2). Fiber-like structures in the electron microscope image obtained from the membrane of the cell culture insert, may be a form of intercellular channels known as TnTs (12). Besides TnTs, further changes in the cell phenotype were observed subsequent to co-culture of rabbit NPCs cultured in the porous membrane insert system with MSCs revealing higher proliferation and proteoglycan synthesis by NPCs (2). Formation of TnTs by human MSCs with other cell types was observed in the co-culture of MSCs with vascular smooth muscle cells (23). TnTs in the system promoted the exchange of mitochondria and increased the proliferation of MSCs initiated by contact with vascular smooth muscle cells (23). In the present study TnT-like structures are shown, which form cytoplasmic channels observed earlier in other cell types, including MSCs. However, the reason for the hydrophilic dyes, used in earlier studies of NP-MSC co-cultures, not exchanging as efficiently as the lipophilic dyes remains to be clarified.

Previous studies demonstrated that human cell contact and exchange of cytoplasm components with the use of fluorescent proteins or hydrophilic dyes have been produced $(8,9)$. Paradoxically, this approach did not produce a large double-stained population of cells. When various lipophilic dyes were applied in rat and human NP and MS cell systems the result was different. Thus, lipophilic dyes may only be exchanged with external phospholipids of the plasma membrane. Since experiments with cytoplasm soluble dyes or fluorescent proteins exhibited a small fraction of double-stained cells, this demonstrates that these dyes are not efficiently packed into MVs or TnTs. The cellular cargo is selectively packed into the MVs of three classes: apoptotic bodies, ectosomes and exosomes (24). The TnTs are plasma membrane protrusions that connect the cytoplasms of communicating cells (12). In addition to enabling the relocation of organelles, TnTs are involved in $\mathrm{Ca}^{+}$fluxes between cells (12). As the hydrophilic dyes are not efficiently transported in co-culture systems, this means that TnTs are not as important for the intercellular transport of cytosol components as for lipid components. Another, although less likely hypothesis, is the selection on entry to these structures, a type of 'gate control'. Further studies are required into this hypothesis. The existence of TnTs in vivo has been documented for certain cell 
types, therefore TnTs between NPCs might enable contact of these remote cells in the structure of NP (12).

Co-culture of DiO-stained NP and DiD-stained MSCs and invert dye DiD-stained NP and DiO-stained MSCs revealed that the exchange of dyes is asymmetrical. Lipophilic dye exchange has been studied in rabbit and human NPCs and MSC co-culture systems $(4,10)$. In rabbit culture, PKH26 (red) and PKH67 (green) lipophilic dyes were used. Subsequent to a two-week incubation period on alginate beads $42 \%$ of the cells were double-stained (4). A flow cytometric analysis revealed that the exchange of dyes between rabbit NPCs and MSCs is balanced and the cytometric diagram was less asymmetrical (in relation to a $45^{\circ}$ axis) than our diagram, nevertheless, it is probable that the MSCs secreted more PKH26 due to fewer PHK67-stained NPCs observed (4). The co-culture resulted in the generation of a double-stained cell population characterized by increased Col2al and Acan mRNAs levels. Double nucleolar hybrids were also detected (4).

In comparison to former results, the experiments of this study demonstrated a more rapid flow of DiO and DiD between NPCs and MSCs and after 8 days the majority of the cells were double-stained. Comparing our flow cytometric results from direct co-culture experiments: $\mathrm{NP} / \mathrm{DiO}$ and MSC/DiD vs. MSC/DiO and NP/DiD both diagrams were asymmetrical but not identical. The asymmetrical diagrams may reflect combined effect of various properties of $\mathrm{DiO}$ and DiD and reveals more efficient flow of both fluorescent lipophilic dyes DiO and DiD from MSCs toward NPCs in the direct systems (25). Asymmetrical secretion of lipophylic dye $\mathrm{DiO}$ was already observed in co-culture of renal tubular cells and MSCs (26). In the indirect system we did not observe this preferred direction of the DiD flow from MSCs toward NPCs. We concluded that direct contact and TnT formation enables more rapid flow of dyes from MSCs toward NPCs.

Based on previous results of experiments performed in NPCs and MSCs co-culture systems in which only $<1 \%$ of cells formed hybrids, a hypothesis of cell fusion was abandoned as a rare event (27). A hypothesis of transient, short-term fusion and fission cannot be excluded nor confirmed based on the present and previous results. A hypothesis of the presence of TnTs was confirmed by the present results where double-staining of NPCs and MSCs with DiO and DiD was applied and followed by the observation under a confocal microscope. A hypothesis of hemifusion of membranes is markedly supported by the current results but not proven. The hemifusion hypothesis was able to explain the conflicting results. In previous studies $\sim 1 \%$ of co-cultured NPCs and MSCs were double-stained (fused) following staining with hydrophilic dyes or fluorescent proteins $(8,10,28)$. By contrast, in similar culture systems in which lipophilic dyes were applied, up to $42 \%$ of double-stained cells were observed. Our cytometric measurements revealed $>50 \%$ of cells were double-stained by $\mathrm{DiO}$ and $\mathrm{DiD}$. Hemifusion may concern both MVs in the insert system and whole cells in the one-surface system where only membrane phospholipids are exchanged without pore formation (29). The transfer of membrane lipids and lipophilic dyes was higher compared with that of the membrane proteins (21). Membrane lipids, gangliosides and sphinogosine-phosphate, may promote differentiation of MSCs independently or with growth factors (30-32). Independent cell-cell membrane contact may be a factor initi- ating the potential changes which are a functional determinant of MSC differentiation and cell function (33).

However, issues concerning the nature of the information which is transported via MVs and TnTs remain. The most efficient way of chondrocyte-like NP phenotype induction from MSCs remains under debate and requires additional studies concerning phenotype induction.

Hybrids in the co-cultured cells account $<1 \%$ and it is likely that they do not contribute to the net phenotype of the cell co-culture $(8,10)$. However, no clear rules emerged as to which phenotype may predominate in hybrids (34). The mechanisms by which one phenotype predominates over another in such hybrids remains poorly understood. The same consideration may be applied in co-culture systems. The resulting phenotype of the two co-cultured cells is unknown and unpredictable. Degenerated NPCs co-cultured with MSCs have a higher cell division rate and gene expression. MSCs express cartilage-specific genes. No theory predicting the phenotype of cells which were maintained with other cells in co-culture has been suggested. In general, it was concluded that the 'contagious phenotype theory' predominates in the description of the results of these investigations. These approaches have a default assumption that MSCs 'learn' their phenotype from NPCs, and promote cell division in NPCs. However, the manner in which the NP phenotype can be mimicked by MSCs and whether or not this method is safe with regard to possible chromosomal rearrangement due to hybrid formation remain to be determined (22).

\section{Acknowledgements}

This study was supported by a National Science Centre grant, no. N N 403600538. We would like to thank Ms Beata Raczak and Bogumila Ratajczak for their indispensable help during the preparation of this manuscript.

\section{References}

1. Roberts S, Evans H, Trivedi J and Menage J: Histology and pathology of the human intervertebral disc. J Bone Joint Surg Am 88 Suppl 2: 10-14, 2006.

2. Yamamoto Y, Mochida J, Sakai D, et al: Upregulation of the viability of nucleus pulposus cells by bone marrow-derived stromal cells: significance of direct cell-to-cell contact in coculture system. Spine (Phila Pa 1976) 29: 1508-1514, 2004.

3. Wei A, Chung SA, Tao H, et al: Differentiation of rodent bone marrow mesenchymal stem cells into intervertebral disc-like cells following coculture with rat disc tissue. Tissue Eng Part A 15: 2581-2595, 2009.

4. Niu CC, Yuan LJ, Lin SS, Chen LH and Chen WJ: Mesenchymal stem cell and nucleus pulposus cell coculture modulates cell profile. Clin Orthop Relat Res 467: 3263-3272, 2009.

5. Le Visage C, Kim SW, Tateno K, Sieber AN, Kostuik JP and Leong KW: Interaction of human mesenchymal stem cells with disc cells: changes in extracellular matrix biosynthesis. Spine (Phila Pa 1976) 31: 2036-2042, 2006.

6. Richardson SM, Walker RV, Parker S, et al: Intervertebral disc cell-mediated mesenchymal stem cell differentiation. Stem Cells 24: 707-716, 2006.

7. Yang SH, Wu CC, Shih TT, Sun YH and Lin FH: In vitro study on interaction between human nucleus pulposus cells and mesenchymal stem cells through paracrine stimulation. Spine (Phila $\mathrm{Pa}$ 1976) 33: 1951-1957, 2008.

8. Vadalà G, Studer RK, Sowa G, et al: Coculture of bone marrow mesenchymal stem cells and nucleus pulposus cells modulate gene expression profile without cell fusion. Spine (Phila Pa 1976) 33: 870-876, 2008. 
9. Strassburg S, Richardson SM, Freemont AJ and Hoyland JA Co-culture induces mesenchymal stem cell differentiation and modulation of the degenerate human nucleus pulposus cell phenotype. Regen Med 5: 701-711, 2010.

10. Strassburg S, Hodson NW, Hill PI, Richardson SM and Hoyland JA: Bi-directional exchange of membrane components occurs during co-culture of mesenchymal stem cells and nucleus pulposus cells. PLoS One 7: e33739, 2012.

11. Gerdes HH, Rustom A and Wang X: Tunneling nanotubes, an emerging intercellular communication route in development. Mech Dev 131: 381-387, 2013.

12. Abounit $\mathrm{S}$ and Zurzolo $\mathrm{C}$ : Wiring through tunneling nanotubes - from electrical signals to organelle transfer. J Cell Sci 125: 1089-1098, 2012.

13. Horwitz EM, Le Blanc K, Dominici M, et al: Clarification of the nomenclature for MSC: The International Society for Cellular Therapy position statement. Cytotherapy 7: 393-395, 2005.

14. Huang S, Tam V, Cheung KM, et al: Stem cell-based approaches for intervertebral disc regeneration. Curr Stem Cell Res Ther 6: 317-326, 2011.

15. Xie LW, Fang H, Chen AM and Li F: Differentiation of rat adipose tissue-derived mesenchymal stem cells towards a nucleus pulposus-like phenotype in vitro. Chin J Traumatol 12: 98-103, 2009.

16. Feng G, Jin X, Hu J, et al: Effects of hypoxias and scaffold architecture on rabbit mesenchymal stem cell differentiation towards a nucleus pulposus-like phenotype. Biomaterials 32: 8182-8189, 2011

17. Gantenbein-Ritter B, Benneker LM, Alini M and Grad S Differential response of human bone marrow stromal cells to either TGF- $\beta$ (1) or rhGDF-5. Eur Spine J 20: 962-971, 2011.

18. Morigele M, Shao Z, Zhang Z, et al: TGF- $\beta 1$ induces a nucleus pulposus-like phenotype in Notch 1 knockdown rabbit bone marrow mesenchymal stem cells. Cell Biol Int 37, 820-825, 2013.

19. Yuan M, Leong KW and Chan BP: Three-dimensional culture of rabbit nucleus pulposus cells in collagen microspheres. Spine J 11: 947-960, 2011

20. Leckie SK, Sowa GA, Bechara BP, et al: Injection of human umbilical tissue-derived cells into the nucleus pulposus alters the course of intervertebral disc degeneration in vivo. Spine J 13 263-272, 2013

21. Niu X, Gupta K, Yang JT, Shamblott MJ and Levchenko A Physical transfer of membrane and cytoplasmic components as a general mechanism of cell-cell communication. J Cell Sci 122 600-610, 2009
22. Oren-Suissa M and Podbilewicz B: Evolution of programmed cell fusion: common mechanisms and distinct functions. Dev Dyn 239: 1515-1528, 2010.

23. Vallabhaneni KC, Haller H and Dumler I: Vascular smooth muscle cells initiate proliferation of mesenchymal stem cells by mitochondrial transfer via tunneling nanotubes. Stem Cells Dev 21: 3104-3113, 2012.

24. Lee TH, D'Asti E, Magnus N, Al-Nedawi K, Meehan B and Rak J: Microvesicles as mediators of intercellular communication in cancer - the emerging science of cellular 'debris'. Semin Immunopathol 33: 455-467, 2011.

25. Chazotte B: Labeling membranes with carbocyanine dyes (Dils) as phospholipid analogs. Cold Spring Harb Protoc 2011: pdb prot5555, 2011

26. Plotnikov EY, Khryapenkova TG, Galkina SI, Sukhikh GT and Zorov DB: Cytoplasm and organelle transfer between mesenchymal multipotent stromal cells and renal tubular cells in co-culture. Exp Cell Res 316: 2447-2455, 2010.

27. Chen EH and Olson EN: Unveiling the mechanisms of cell-cell fusion. Science 308: 369-373, 2005.

28. Ferrand J, Noël D, Lehours P, et al: Human bone marrow-derived stem cells acquire epithelial characteristics through fusion with gastrointestinal epithelial cells. PLoS One 6: e19569, 2011.

29. Chernomordik LV and Kozlov MM: Membrane hemifusion: crossing a chasm in two leaps. Cell 123: 375-382, 2005.

30. Nincheri P, Luciani P, Squecco R, et al: Sphingosine 1-phosphate induces differentiation of adipose tissue-derived mesenchymal stem cells towards smooth muscle cells. Cell Mol Life Sci 66: $1741-1754,2009$

31. Yang HJ, Jung KY, Kwak DH, et al: Inhibition of ganglioside GD1a synthesis suppresses the differentiation of human mesenchymal stem cells into osteoblasts. Dev Growth Differ 53: 323-332, 2011.

32. Yang L, Chang N, Liu X, et al: Bone marrow-derived mesenchymal stem cells differentiate to hepatic myofibroblasts by transforming growth factor- $\beta 1$ via sphingosine kinase/sphingosine 1-phosphate (S1P)/S1P receptor axis. Am J Pathol 181: 85-97, 2012.

33. Sundelacruz S, Levin M and Kaplan DL: Membrane potential controls adipogenic and osteogenic differentiation of mesenchymal stem cells. PLoS One 3: e3737, 2008.

34. Essentials of Stem Cell Biology. Lanza R, Gearhart J, Hogan B, Melton D, Pedersen R, Donnall Thomas E, Thomson AJ, Wilmut I (eds). Academic Press, pp105-110, 2009. 